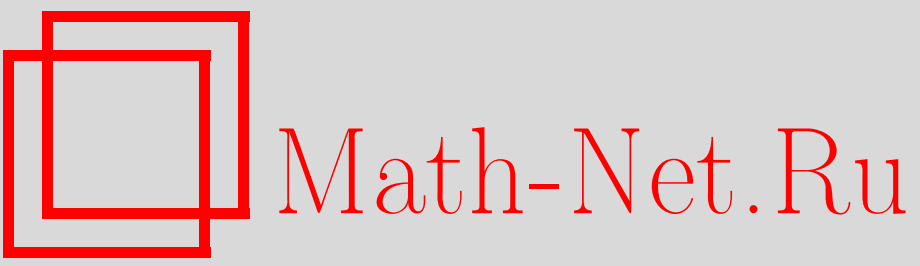

Д. А. Славнов, Статистико-алгебраический подход к квантовой механике, ТМФ, 2001, том 129, номер 1, 87-102

DOI: https://doi.org/10.4213/tmf521

Использование Общероссийского математического портала Math-Net.Ru подразумевает, что вы прочитали и согласны с пользовательским соглашением

http://www.mathnet.ru/rus/agreement

Параметры загрузки:

IP: 54.198 .64 .247

26 апреля 2023 г., 13:58:08 
ТЕОРЕТИЧЕСКАЯ

И МАТЕМАТИЧЕСКАЯ

ФИЗИКА

Том 129, № 1

октябрь, 2001

(C) 2001 г.

Д. А. Славнов*

\title{
СТАТИСТИКО-АЛГЕБРАИЧЕСКИЙ ПОДХОД К КВАНТОВОЙ МЕХАНИКЕ
}

\begin{abstract}
Предложена схема построения квантовой механики, в которой гильбертово пространство и линейные операторы не являются первичными элементами теории. Вместо этого рассматривается некоторый вариант алгебраического подхода. В качестве первичных составляющих используются элементы некоммутативной алгебры (наблюдаемые) и нелинейные функционалы на этой алгебре (физические состояния). Последние ассоциируются с результатами единичного измерения. В качестве квантовых состояний стандартной квантовой механики предлагается рассматривать некоторые ансамбли физических состояний. Показано, что в такой схеме может быть воспроизведен математический аппарат стандартной квантовой механики в полном объеме.
\end{abstract}

\section{1. ВВЕДЕНИЕ}

Уже давно среди большинства физиков утвердилось мнение, что в знаменитом споре Бора с Эйнштейном об основах квантовой механики прав был Бор. Однако, возможно, дело обстоит не так просто. Основной тезис Эйнштейна был таков [1]: в современном виде квантовая теория является вполне корректной статистической теорией ансамблей физических систем, но не теорией отдельных элементарных процессов. В этом смысле она не является полной теорией (по этому поводу см. также [2]).

В некотором смысле стандартная квантовая механика (квантовая механика Бора, Гейзенберга, Дирака, фон Неймана) подобна термодинамике. В обоих этих разделах физики многие основные понятия связаны с глобальными характеристиками некоторых ансамблей физических объектов. В термодинамике такими понятиями являются термодинамические потенциалы, в квантовой физике - векторы состояния.

В общем случае, зная вектор состояния, нельзя предсказать во всех деталях поведение отдельного физического объекта. Приходится ограничиваться вероятностными суждениями или давать усредненную характеристику ансамбля, состояшего из большого числа таких объектов.

В принципе законы термодинамики можно сформулировать, не ссылаясь на свойства отдельных членов ансамбля. Однако известно, насколько плодотворной оказалась идея

* Московский государственный университет, Москва, Россия 
рассматривать основные термодинамические величины как статистические средние более элементарных величин. Соответственно оказалось очень полезно считать законы термодинамики не аксиомами, а следствиями законов статистической физики, которая основывается на теории вероятностей и на совокупности законов, управляющих поведением элементарных физических объектов.

В настоящей статье делается попытка осушествить нечто подобное в случае квантовой механики. Однако в отличие от классической термодинамики, здесь не предполагается, что законы для отдельных элементов рассматриваемых ансамблей тождественны законам классической механики. Вместо этого используются некоторые основные положения алгебраического подхода к квантовой теории. Этот подход уже достаточно хорошо разработан и описан в ряде монографий (см., например, [3]- [5]).

Центральную роль в нашем рассмотрении будут играть некоторые нелинейные функционалы (физические состояния), определенные на алгебрах наблюдаемых. Именно эти функционалы ассоциируются с отдельными элементарными процессами. Привычным квантовым состояниям стандартной квантовой механики будут сопоставлены ансамбли таких функционалов. В таком своеобразном виде в настоящей статье делается попытка продвинуться в направлении, которое отстаивал Эйнштейн.

\section{2. АЛГЕБРЫ НАБЛЮДАЕМЫХ И ФИЗИЧЕСКИЕ СОСТОЯНИЯ}

В свете вышесказанного при построении квантовой теории предлагается не класть в ее основу аксиому стандартной квантовой механики: наблюдаемым величинам соответствуют самосопряженные линейные операторы в гильбертовом пространстве. Это утверждение вряд ли может претендовать на роль очевидного факта, что очень желательно для аксиомы.

Вместо этого утверждения примем гораздо более слабую аксиому алгебраического подхода к квантовой теории: наблюдаемым величинам соответствуют элементы некоторой алгебры. Это означает только, что наблюдаемые величины можно умножать на числа, складывать и перемножать между собой. Так как все наблюдаемые величины действительны, то, вероятно, можно ограничиться действительной алгеброй. На эту роль может претендовать йорданова алгебра наблюдаемых (см. [3]). Однако в этой алгебре операция умножения определяется сравнительно сложно и отсутствует свойство ассоциативности. В связи с этим кажется оправданным выйти за рамки непосредственно наблюдаемых величин и рассматривать их комплексные комбинации, которые в дальнейшем будут называться динамическими величинами.

В соответствии с вышеизложенным примем первый постулат.

Динамическим величинам соответствуют әлементы инволютивной ассоциативной (в общем случае) некоммутативной алгебры $\mathfrak{A}$, удовлетворяющей условиям: 1) для каждого әлемента $\widehat{R} \in \mathfrak{A}$ найдется әрмитов әлемент $\hat{A} \quad\left(\hat{A}^{*}=\hat{A}\right)$ такой, что $\left.\widehat{R}^{*} \widehat{R}=\hat{A}^{2} ; 2\right)$ если $\widehat{R}^{*} \widehat{R}=0$, mо $\widehat{R}=0$.

Здесь и везде далее предполагается, что в определение алгебры включено требование наличия единичного элемента $\hat{I}$. Наблюдаемым величинам соответствуют эрмитовы элементы алгебры $\mathfrak{A}$. Их множество обозначим $\mathfrak{A}_{+}$. Будем считать, что все элементы 
действительного линейного пространства $\mathfrak{A}_{+}$соответствуют наблюдаемым. Строго говоря, это не всегда справедливо, так как могут существовать правила суперотбора. Но эту проблему в данной статье мы рассматривать не будем.

Bторой постулат позаимствуем непосредственно из стандартной квантовой механики.

Наблюдаемие $\hat{A}$ и $\widehat{B}$ совместно измерими, когда $[\hat{A}, \widehat{B}]=0$.

Обычно говорят, что такие наблюдаемые одновременно измеримы с любой степенью точности, хотя факт одновременности не всегда играет определяюшую роль. Кроме того, одновременность не является релятивистски-инвариантным понятием. Более четко понятие совместимости можно определить следуюшим образом. Пусть с наблюдаемыми $\hat{A}$ и $\widehat{B}$ проводится следуюшая последовательность измерений: сначала измеряется наблюдаемая $\hat{A}$, затем наблюдаемая $\widehat{B}$, потом повторно наблюдаемая $\hat{A}$ и, наконец, снова наблюдаемая $\widehat{B}$. Наблюдаемые $\hat{A}$ и $\widehat{B}$ являются совместно измеримыми, если в идеальном эксперименте с любой степенью точности результаты повторного измерения каж дой из наблюдаемых совпадают с результатами первичного измерения соответствуюшей наблюдаемой. Здесь использование понятий "сначала" и "затем" предполагает, что события отделены времениподобным интервалом.

В связи со вторым постулатом в дальнейшем сушественную роль будут играть коммутативные подалгебры алгебры $\mathfrak{A}$. Обозначим через $\mathfrak{Q}\left(\mathfrak{Q} \equiv\{\widehat{Q}\} \in \mathfrak{A}_{+}\right)$максимальную действительную коммутативную подалгебру алгебры $\mathfrak{A}$. Это подалгебра совместно измеримых наблюдаемых. Если алгебра $\mathfrak{A}$ коммутативна (алгебра классических динамических величин), то такая подалгебра одна. Если алгебра $\mathfrak{A}$ некоммутативна (алгебра квантовых динамических величин), то таких подалгебр много. Более того, множество, элементами которого являются подалгебры $\mathfrak{Q}$, имеет мощность континуума. Действительно, уже для алгебры $\mathfrak{A}$ с двумя некоммутируюшими эрмитовыми образуюшими $\hat{A}_{1}$ и $\hat{A}_{2}$ для каждого $\alpha$ действительная алгебра $\mathfrak{Q}_{\alpha}$ с образуюшей $\widehat{B}(\alpha)=\hat{A}_{1} \cos \alpha+\hat{A}_{2} \sin \alpha$ будет алгеброй типа $\mathfrak{Q}$.

Напомним определение спектра $\sigma(\hat{A} ; \mathfrak{A})$ элемента $\hat{A}$ алгебры $\mathfrak{A}$. Число $\lambda$ является точкой спектра элемента $\hat{A}$ тогда и только тогда, когда элемент $\hat{A}-\lambda \hat{I}$ не имеет обратного в алгебре $\mathfrak{A}$.

Вообще говоря, один и тот же элемент может иметь разные спектры в алгебре и ее подалгебре. Однако для алгебр типа $\mathfrak{Q}$ справедливо (см. [6]) утверждение: $\sigma(\widehat{Q} ; \mathfrak{Q})=$ $\sigma(\widehat{Q} ; \mathfrak{A})$ для любого $\widehat{Q} \in \mathfrak{Q}$.

Эрмитовы элементы алгебры $\mathfrak{A}$ являются латентной формой наблюдаемых величин. Явной формой наблюдаемой должно быть некоторое число. Иными словами, для задания явной формы наблюдаемых на эрмитовых элементах алгебры $\mathfrak{A}$ должен быть задан некоторый функционал $\varphi: \varphi(\hat{A})=A$ - действительное число.

Физически латентная форма наблюдаемой $\hat{A}$ становится явной в результате измерения. Это означает, что функционал $\varphi(\hat{A})$ должен определять значение наблюдаемой $\hat{A}$, которое могло бы получиться в конкретном (индивидуальном) измерении. Этот функционал назовем физическим состоянием рассматриваемого квантового объекта.

В индивидуальном эксперименте могут быть измерены только взаимно коммутирую- 
щие элементы. При этом сумме и произведению наблюдаемых должны соответствовать сумма и произведение результатов измерений: $\hat{A}_{1}+\hat{A}_{2} \rightarrow A_{1}+A_{2}, \hat{A}_{1} \hat{A}_{2} \rightarrow A_{1} A_{2}$.

Воспользуемся следующим определением (см. [6]). Пусть $\mathfrak{B}$ - комплексная (действительная) коммутативная алгебра и $\varphi$ - линейньй функционал на $\mathfrak{B}$. Если

$$
\varphi\left(\widehat{B}_{1} \widehat{B}_{2}\right)=\varphi\left(\widehat{B}_{1}\right) \varphi\left(\widehat{B}_{2}\right)
$$

для всех $\widehat{B}_{1}, \widehat{B}_{2} \in \mathfrak{B}$, то функционал $\varphi$ называется комплексным (действительным) гомоморфизмом на алгебре $\mathfrak{B}$. Функционал, удовлетворяющий равенству $(1)$, называется также мультипликативным функционалом.

Примем третий постулат.

Физическое состояние конкретного квантового обвекта задается функчионалом $\varphi(\hat{A}) \quad\left(\hat{A} \in \mathfrak{A}_{+}\right)$, сужение которого на любую подалгебру типа $\mathfrak{Q}$ является действительным гомоморфизмом.

Определенный таким образом функционал $\varphi(\hat{A})$ обладает следующими свойствами (см. [6]):

$$
\begin{aligned}
& \varphi(0)=0 ; \\
& \varphi(\hat{I})=1 ; \\
& \varphi\left(\hat{A}^{2}\right) \geqslant 0 ; \\
& \text { если } \lambda=\varphi(\hat{A}), \text { то } \lambda \in \sigma(\hat{A} ; \mathfrak{A}) ; \\
& \text { если } \lambda \in \sigma(\hat{A} ; \mathfrak{A}), \text { то } \lambda=\varphi(\hat{A}) \text { для некоторого } \varphi(\hat{A}) .
\end{aligned}
$$

Все эти свойства прекрасно согласуются с результатами индивидуальных экспериментов.

Представляется естественным наложить на алгебру $\mathfrak{A}$ дополнительное требование, заключающееся в том, что для любых двух наблюдаемых существует эксперимент (т.е. функционал $\varphi$ ), который их разделяет. В соответствии с этим примем четвертый поcmyлат:

$\varphi\left(\hat{A}_{1}\right)=\varphi\left(\hat{A}_{2}\right)$ для всех $\varphi$ тогда и только тогда, когда $\hat{A}_{1}=\hat{A}_{2}$.

Заметим, что в стандартной квантовой механике делается более сильное предположение: наблюдаемые совпадают, если совпадают все их средние значения. В стандартной квантовой механике постулируется также, что результат индивидуального измерения наблюдаемой принадлежит ее спектру. В предлагаемом подходе это получается автоматически (свойства (2г) и (2д)).

Свойством линейности обладают только сужения функционалов $\varphi$ на подалгебры $\mathfrak{Q}$, а на множестве $\mathfrak{A}_{+}$физические состояния не являются линейными функционалами.

Множество всех физических состояний рассматриваемой физической системы уместно назвать фазовым пространством этой системы, т.е. точкой фазового пространства $M$ является (нелинейный) функционал $\varphi$ на множестве $\mathfrak{A}_{+}$. 
Так же как в случае классического фазового пространства, точка в $M$ может быть задана с помошью набора действительных чисел (координат). Этот набор можно построить следующим образом. Выбираем некоторую подалгебру $\mathfrak{Q}_{1}$ (типа $\mathfrak{Q}$ ). Пусть $G\left(\mathfrak{Q}_{1}\right)$ - набор образующих алгебры $\mathfrak{Q}_{1}$. Функционал $\varphi$ отображает набор $G\left(\mathfrak{Q}_{1}\right)$ в набор $S_{1}$ действительных чисел (точек спектра алгебры $\left.\mathfrak{A}\right)$ :

$$
G\left(\mathfrak{Q}_{1}\right) \stackrel{\varphi}{\longrightarrow} S_{1} .
$$

Далее выбираем другую подалгебру $\mathfrak{Q}_{2}$. Если $G\left(\mathfrak{Q}_{1}\right) \cap \mathfrak{Q}_{2} \equiv \widetilde{G}_{1} \neq \varnothing$, элементы набора $\widetilde{G}_{1}$ возьмем в качестве поднабора образуюших алгебры $\mathfrak{Q}_{2}$. Произвольным образом дополняем этот поднабор до полного набора $G\left(\mathfrak{Q}_{2}\right)$ образующих алгебры $\mathfrak{Q}_{2}$. Аналогично формуле (3)

$$
G\left(\mathfrak{Q}_{2}\right) \stackrel{\varphi}{\longrightarrow} S_{2} \text {. }
$$

Затем строим образующие подалгебры $\mathfrak{Q}_{3}$, включая в их состав элементы из набора $\left[G\left(\mathfrak{Q}_{1}\right) \cup G\left(\mathfrak{Q}_{2}\right)\right] \cap \mathfrak{Q}_{3}$. И так далее.

Если алгебра $\mathfrak{A}$ коммутативна (классическая физическая система), то процесс оборвется на первом этапе, и мы получим координаты обычного классического фазового пространства. Если алгебра $\mathfrak{A}$ некоммутативная, то подалгебр $\mathfrak{Q}_{i}$ будет бесконечно много. Процесс никогда не оборвется. Набор координат $\bigcup_{i} S_{i}$, определяющий отдельную точку фазового пространства, будет бесконечным. Каждая координата является действительной точкой спектра алгебры $\mathfrak{A}$.

Видно, что структура фазового пространства квантовой системы аналогична структуре фазового пространства классического действительного поля. Поэтому идеи Эйнштейна о том, что одной из составляющих фундамента полной квантовой теории должны быть классические поля обшей теории относительности, возможно, не совсем беспочвенны.

\section{3. КВАНТОВЫЕ СОСТОЯНИЯ}

Теперь введем конструкцию, которая соответствует чистому состоянию в стандартной квантовой механике. Функционал $\varphi$ отображает множество $\mathfrak{Q}=\{\widehat{Q}\}$ (максимальную коммутативную подалгебру) в множество действительных чисел:

$$
\{\widehat{Q}\} \stackrel{\varphi}{\longrightarrow}\{Q=\varphi(\widehat{Q})\} .
$$

Для разных функционалов $\varphi_{i}, \varphi_{j}$ множества $\left\{\varphi_{i}(\widehat{Q})\right\},\left\{\varphi_{j}(\widehat{Q})\right\}$ могут различаться, а могут совпадать. Если для всех $\widehat{Q} \in\{\widehat{Q}\}$ справедливо $\varphi_{i}(\widehat{Q})=\varphi_{j}(\widehat{Q})=Q$, то функционалы $\varphi_{i}$ и $\varphi_{j}$ назовем $\{Q\}$-эквивалентными. Пусть $\{\varphi\}_{Q}$ множество всех физических состояний, которым соответствуют $\{Q\}$-эквивалентные функционалы. Множество $\{\varphi\}_{Q}$ назовем квантовым состоянием и обозначим $\Psi_{Q}$. Ясно, что для однозначной фиксации $\Psi_{Q}$ вовсе не обязательно задавать множество значений для всех наблюдаемых $\{\widehat{Q}\}$. Достаточно ограничиться значениями функционалов для образующих алгебры $\mathfrak{Q}$.

Квантовому состоянию $\Psi_{Q}$ соответствует поверхность (бесконечномерная) в фазовом пространстве квантовой системы. 
Экспериментально о функционале $\varphi$ полной информации получить нельзя. Действительно, в одном эксперименте мы можем измерить только взаимно коммутирующие наблюдаемые. Все эти наблюдаемые являются элементами некоторой подалгебры $\mathfrak{Q}$. Но все $\{Q\}$-эквивалентные функционалы имеют одинаковые значения на любой наблюдаемой $\widehat{Q} \in\{\widehat{Q}\}$. Поэтому с помошью проведенного эксперимента мы не сможем отличить один функционал $\varphi$ от другого, если они оба принадлежат набору $\{\varphi\}_{Q}$. Попытка для того же физического объекта измерить наблюдаемую $\hat{A} \notin\{\widehat{Q}\}$ в последующем эксперименте не поможет. В силу второго постулата этот эксперимент даст искаженные сведения о значении $\varphi(\hat{A})$ для функционала, соответствующего физическому состоянию, в котором исследуемый объект находился до первого эксперимента.

Можно было бы надеяться измерить наблюдаемую $\hat{A} \notin\{\widehat{Q}\}$ для другого объекта, находящегося в том же физическом состоянии. Однако для этого предварительно надо удостовериться, что этот объект находится в том же физическом состоянии, что первый. А это нельзя сделать, не проведя для обоих объектов измерения всех наблюдаемых. Это невозможно в силу того же второго постулата.

О функционале $\varphi$ мы можем экспериментально получить информацию, достаточную для того, чтобы убедиться, что он принадлежит к некоторому множеству $\{\varphi\}_{Q}$, т.е. квантовому состоянию. Для этого достаточно провести измерения взаимно коммутируюших наблюдаемых, можно ограничиться только соответствующими образующими. В принципе это можно проделать в одном эксперименте.

Строго говоря, это справедливо только в том случае, когда в рассматриваемой физической системе нет одинаковых частиц. Дело в том, что измерительный прибор не различает, какая из одинаковых частиц в него попала. Поэтому чтобы сохранить справедливость утверждения предыдущего абзаца, необходимо несколько обобщить определение квантового состояния.

Пусть физическая система содержит одинаковые частицы. Пусть $\{\varphi\}_{Q}$ - множество $\{Q\}$-эквивалентных функционалов. Функционал $\tilde{\varphi}$ назовем слабо $\{Q\}$-эквивалентным функционалам $\{\varphi\}_{Q}$, если сушествует такое множество $\left\{\widehat{Q}^{\prime}\right\}$, на котором функционал $\tilde{\varphi}$ принимает те же значения, которые функционалы $\{\varphi\}_{Q}$ принимают на множестве $\{\widehat{Q}\}$. Множество $\left\{\widehat{Q}^{\prime}\right\}$ должно являться отображением множества $\{\widehat{Q}\}$, при котором наблюдаемые, относящиеся к одной из одинаковых частиш, заменяются соответствуюшими наблюдаемыми, относящимися к другой.

Назовем теперь квантовым состоянием $\Psi_{Q}$ множество всех слабо $\{Q\}$-эквивалентных функционалов. Далее будем использовать обозначение $\{\varphi\}_{Q}$ для множества всех слабо $\{Q\}$-эквивалентных функционалов.

Таким образом, о физическом состоянии конкретного физического объекта мы принципиально не можем иметь полной информации. Максимальная информация, которую с помошью измерений можно получить о физическом объекте, позволяет только констатировать, что он находится в определенном квантовом состоянии.

В действительности иногда удается получить несколько бо́льшую информацию о физическом состоянии. Например, в описанном в раздел 4 эксперименте с помощью измерения проекции спина частицы $A$ на ось $z$ можно определить проекцию спина на эту ось 
для частицы $B$ (косвенное измерение). Одновременно с этим можно непосредственно измерить проекцию спина частицы $B$ на ось $x$. Однако в любом случае нельзя экспериментально найти $\varphi(\hat{A})$ для всех наблюдаемых.

Из сказанного выше следует, что о начальном физическом состоянии конкретного квантового объекта мы всегда имеем неполную информацию. Поэтому даже при строго детерминистских законах эволюции мы можем делать только вероятностные предсказания о значениях большинства наблюдаемых. Ситуация вполне аналогична той, которая имеет место в статистической физике.

Квантовое фазовое пространство имеет мощность континуума. Мы не обладаем специальными возможностями организовать эксперимент так, чтобы в нем фигурировало наперед заданное физическое состояние. Поэтому вероятность того, что изучаемый квантовый объект находится в наперед заданном физическом состоянии, равна нулю. Отсюда следует, что равна нулю вероятность того, что в двух разных экспериментах мы столкнемся с двумя одинаковыми физическими состояниями. Более того, равна нулю вероятность встречи с двумя одинаковыми физическими состояниями в любой конечной и даже счетной последовательности экспериментов. Это, конечно, не означает, что такое событие является невозможным. Тем не менее мы можем считать, что в разных экспериментах мы всегда имеем дело с разными физическими состояниями.

Воспользуемся некоторыми положениями теории вероятностей. Будем рассматривать фазовое пространство (множество физических состояний) в качестве генеральной совокупности, а каждый эксперимент по измерению наблюдаемой-как испытание (термин теории вероятностей). В качестве события $\tilde{A}$ будем рассматривать эксперимент, в котором измеренное значение наблюдаемой $\hat{A}$ не больше $\tilde{A}$, т.е. $\varphi(\hat{A})=A \leqslant \tilde{A}$.

Это событие не является безусловным. В квантовой теории имеется специфическое условие: одно испытание не может быть событием для измерения двух некоммутируюших наблюдаемых. Вероятность события $\tilde{A}$ определяется структурой фазового пространства и указанным условием. Пусть эта вероятность равна $P(\tilde{A})$.

Рассмотрим выборку из $n$ взаимно независимых случайных испытаний. По определению вероятность появления события $\tilde{A}$ в каждом из этих испытаний равна $P(\tilde{A})$. Пусть событие $\tilde{A}$ появляется в этой выборке $k_{n}$ раз. Тогда по определению $k_{n} / n$-вероятность появления события $\tilde{A}$ в выборке. По теореме Бернулли (см. [7]) при $n \rightarrow \infty$ величина $k_{n} / n$ сходится по вероятности к $P(\tilde{A})$.

Таким образом, величина, к которой по вероятности сходится $k_{n} / n$ при $n \rightarrow \infty$, является вероятностью появления события $\tilde{A}$ в любой счетной выборке из независимых случайных испытаний. Для всех таких выборок эта вероятность одинакова и равна $P(\tilde{A})$. Она определяет вероятностную меру $\mu(\varphi)(\varphi(\hat{A}) \leqslant \tilde{A})$ на любой такой выборке.

Воспользуемся теоремой Хинчина (см. [7]), сформулированной в терминах, которые используются в настоящей статье.

Теорема. Пусть $A_{1}=\varphi_{1}(\hat{A}), \ldots, A_{n}=\varphi_{n}(\hat{A})$ - последовательность значений наблюдаемой $\hat{A}$ в указанной выборке физических состояний. Каждое $A_{i}$ имеет одни и те же распределение $(P(\tilde{A}))$ и конечное математическое ожсидане $\langle A\rangle$. Тогда при $n \rightarrow \infty$ случайная величина $\bar{A}_{n}=\left(A_{1}+\cdots+A_{n}\right) / n$ сходится по вероят- 
ности $\kappa\langle A\rangle$.

Таким образом,

$$
\langle A\rangle=\lim _{n \rightarrow \infty} \mathrm{P}\left\{\frac{1}{n}\left(\varphi_{1}(\hat{A})+\cdots+\varphi_{n}(\hat{A})\right)\right\}=\int d \mu(\varphi) \varphi(\hat{A}),
$$

где интеграл берется по случайной счетной выборке.

Случайную счетную выборку, в которую входят все эксперименты по измерению величины наблюдаемой $\hat{A}$, назовем множеством актуальных состояний для $\hat{A}$ и обозначим $[\varphi]^{\hat{A}}$. Так же определим множество актуальных состояний для наблюдаемой $\widehat{B}$. Пусть $[\hat{A}, \widehat{B}] \neq 0$, тогда наблюдаемые $\hat{A}$ и $\widehat{B}$ должны измеряться в разных экспериментах. Так как множества $[\varphi]^{\hat{A}}$ и $[\varphi]^{\widehat{B}}$ счетные, то вероятность их пересечения равна нулю. Поэтому с вероятностью единица для актуальных состояний дополнительное условие вьполняется автоматически.

В предыдущих рассуждениях под наблюдаемой $\hat{A}$ можно подразумевать совокупность взаимно коммутирующих наблюдаемых, которые измеряются каждый раз в одном эксперименте.

Все рассуждения сохраняются, если на события будут наложены некоторые дополнительные условия. В частности, следующее (представляющее особый интерес): все испытуемые физические состояния принадлежат некоторому квантовому состоянию $\Psi_{Q}=$ $\{\varphi\}_{Q}$.

В этом случае формулу (4) можно переписать следующим образом:

$$
\Psi_{Q}(\hat{A})=\lim _{n \rightarrow \infty} \mathrm{P}\left\{\frac{1}{n}\left(\varphi_{1}(\hat{A})+\cdots+\varphi_{n}(\hat{A})\right)\right\}=\int_{\{\varphi\}_{Q}^{\hat{A}}} d \mu_{Q}(\varphi) \varphi(\hat{A})
$$

где $\{\varphi\}_{Q}^{\hat{A}}=\{\varphi\}_{Q} \cap[\varphi]^{\hat{A}}, \varphi_{i} \in\{\varphi\}_{Q}^{\hat{A}}$.

Формула (5) определяет функционал (квантовое среднее) на множестве $\mathfrak{A}_{+}$. Вся совокупность квантовых экспериментов однозначно свидетельствует в пользу того, что мы должны принять пятый постулат.

Функционал $\Psi_{Q}$ линеен на множестве $\mathfrak{A}_{+}$.

Это значит, что

$$
\Psi_{Q}(\hat{A}+\widehat{B})=\Psi_{Q}(\hat{A})+\Psi_{Q}(\widehat{B})
$$

и в том случае, когда $[\hat{A}, \widehat{B}] \neq 0$.

В стандартной квантовой механике аналогичное утверждение является следствием трех аксиом:

а) каждой наблюдаемой $\hat{A}$ соответствует линейньй оператор $\mathcal{A}$ в некотором гильбертовом пространстве;

б) если наблюдаемым $\hat{A}$ и $\widehat{B}$ соответствуют операторы $\mathcal{A}$ и $\mathcal{B}$, то наблюдаемой $\hat{A}+\widehat{B}$ соответствует оператор $\mathcal{A}+\mathcal{B}$ (одновременная измеримость $\hat{A}$ и $\widehat{B}$ не обязательна); 
в) среднее значение наблюдаемой равняется математическому ожиданию соответствующего оператора.

Ни одно из этих утверждений не следует однозначно из экспериментальных фактов. Другое дело, что они не противоречат всей совокупности экспериментальных данных и позволяют построить прекрасно работающий математический аппарат стандартной квантовой механики.

Любой элемент $\widehat{R}$ алгебры $\mathfrak{A}$ можно однозначно представить в виде $\widehat{R}=\hat{A}+i \widehat{B}$, где $\hat{A}, \widehat{B} \in \mathfrak{A}_{+}$. Поэтому функционал $\Psi_{Q}$ можно продолжить до линейного функционала на алгебре $\mathfrak{A}: \Psi_{Q}(\widehat{R})=\Psi_{Q}(\hat{A})+i \Psi_{Q}(\widehat{B})$.

Пусть $\widehat{R}^{*} \widehat{R}=\widehat{A}^{2} \in\{\widehat{Q}\}\left(\hat{A} \in \mathfrak{A}_{+}\right)$. Если $\varphi \in\{\varphi\}_{Q}$, то $\varphi\left(\widehat{R}^{*} \widehat{R}\right)=A^{2}$. Поэтому

$$
\Psi_{Q}\left(\widehat{R}^{*} \widehat{R}\right)=A^{2}=\left.\varphi\left(\hat{A}^{2}\right)\right|_{\varphi \in\{\varphi\}_{Q}^{\hat{A}}}
$$

Отсюда

$$
\|\widehat{R}\|^{2} \equiv \sup _{Q} \Psi_{Q}\left(\widehat{R}^{*} \widehat{R}\right)=\sup _{\varphi \in[\varphi]^{\hat{A}}} \varphi\left(\hat{A}^{2}\right)>0, \quad \text { если } \widehat{R} \neq 0 .
$$

Так как $\Psi_{Q}$ - положительньй линейньй функционал, то справедливо неравенство Коши-Буняковского-Шварца

$$
\Psi_{Q}\left(\widehat{R}^{*} \widehat{S}\right) \Psi_{Q}\left(\widehat{S}^{*} \widehat{R}\right) \leqslant \Psi_{Q}\left(\widehat{R}^{*} \widehat{R}\right) \Psi_{Q}\left(\widehat{S}^{*} \widehat{S}\right) .
$$

Поэтому для $\|\widehat{R}\|$ выполняются аксиомы нормы элемента $\widehat{R}$ (см. [3]):

$$
\|\widehat{R}+\widehat{S}\| \leqslant\|\widehat{R}\|+\|\widehat{S}\|, \quad\|\lambda \widehat{R}\|=|\lambda| \cdot\|\widehat{R}\|, \quad\left\|\widehat{R}^{*}\right\|=\|\widehat{R}\| .
$$

Потребуем, чтобы алгебра $\mathfrak{A}$ была полна по норме $\|\cdot\|$. Так как $\varphi\left(\left[\hat{A}^{2}\right]^{2}\right)=\left[\varphi\left(\hat{A}^{2}\right)\right]^{2}$, то $\left\|\widehat{R}^{*} \widehat{R}\right\|=\|\widehat{R}\|^{2}$, т.е. алгебра $\mathfrak{A}$ является $C^{*}$-алгеброй. Учитьвая формулу (2б), заключаем, что $\Psi_{Q}$ является положительным линейным функционалом на $C^{*}$-алгебре, удовлетворяюшим условию нормировки $\Psi_{Q}(\hat{I})=1$. Поэтому согласно конструкции Гельфанда-Наймарка-Сигала (см. [3]) функционал $\Psi_{Q}$ канонически порождает гильбертово пространство и представление алгебры $\mathfrak{A}$ линейными операторами в этом гильбертовом пространстве. Иными словами, в предлагаемом подходе можно полностью воспроизвести математический аппарат стандартной квантовой механики.

При желании предыдушие рассуждения можно рассматривать как обоснование аксиом стандартной квантовой механики, необходимых для построения этого аппарата. Заметим, что принятые в настояшей статье постулаты более непосредственно связаны с экспериментом, чем аксиомы стандартной квантовой механики. Такие чисто математические понятия, как векторы гильбертова пространства (волновые функции) и операторы в гильбертовом пространстве, не являются первичными элементами теории в предлагаемом подходе. Они возникают лишь на втором этапе. Первичными элементами являются наблюдаемые и физические состояния, которые непосредственно связаны с результатами эксперимента. Их можно считать связанными с материальной структурой изучаемого квантового объекта и не зависяшими от наблюдателя (см. [8]). 
В противоположность этому квантовые состояния $\Psi_{Q}$ несут в себе некоторьй субъективный элемент. Дело в том, что одно физическое состояние может принадлежать различным множествам $\{\varphi\}_{Q}$ и $\{\varphi\}_{P}$, т.е. $\varphi \in\{\varphi\}_{Q} \cap\{\varphi\}_{P}$, где в $\{\varphi\}_{Q}$ для определения эквивалентности используется набор $\{\widehat{Q}\}$ взаимно коммутирующих наблюдаемых $\widehat{Q}$, а в $\{\varphi\}_{P}$ используются наблюдаемые $\widehat{P} \in\{\widehat{P}\}$, причем наблюдаемые $\widehat{Q}$ и $\widehat{P}$ между собой не коммутируют. Тогда в зависимости от того, какой набор ( $\{\widehat{Q}\}$ или $\{\widehat{P}\})$ наблюдатель выберет (свобода выбора) для классификации, физическое состояние $\varphi$ будет отнесено либо к квантовому состоянию $\Psi_{Q}=\{\varphi\}_{Q}$, либо к квантовому состоянию $\Psi_{P}=\{\varphi\}_{P}$.

\section{4. ПАРАДОКС ЭЙНШТЕЙНА-ПОДОЛЬСКОГО-РОЗЕНА И СКРЫТЫЕ ПАРАМЕТРЫ}

Субъективная составляюшая в квантовом состоянии является первопричиной знаменитого парадокса Эйнштейна-Подольского-Розена [2]. Для его демонстрации Бом [9] предложил следующий эксперимент.

Пусть частица со спином 0 распадается на две частицы $A$ и $B$ со спинами $1 / 2$, которые разлетаются на большое расстояние. Измерим у частицы $A$ проекцию спина на ось $z$. Пусть результатом этого измерения будет $S_{z}(A)$. Тогда, используя закон сохранения, мы можем утверждать, что с вероятностью единица у частицы $B$ будет обнаружена проекция спина на ось $z$, равная $S_{z}(B)=-S_{z}(A)$. Поэтому мы должны считать, что после измерения спина у частицы $A$ частица $B$ будет находиться в квантовом состоянии с определенной проекцией спина на ось $z$, а именно $-S_{z}(A)$. Однако у частишы $A$ мы могли бы измерить проекцию спина на ось $x$. Пусть результат при этом был бы $S_{x}(A)$. Тогда мы могли бы утверждать, что после такого измерения частица $B$ будет находиться в квантовом состоянии с проекцией спина $S_{x}(B)=-S_{x}(A)$.

Таким образом, наблюдатель по своему желанию “загоняет” частицу $B$ либо в квантовое состояние $\{\varphi\}_{-} S_{z}(A)$, либо в квантовое состояние $\{\varphi\}_{-} S_{x}(A)$, никак физически на частицу $B$ не воздействуя.

По мысли авторов статьи [2] такое положение свидетельствует о неполноте квантовой механики, поскольку нарушаются следуюшие требования:

1) каждый элемент физической реальности должен иметь копию в полной физической теории;

2) если без какого-либо возмушения системы мы можем с уверенностью (т.е. с вероятностью единица) предсказать значение физической величины, то, значит, существует элемент реальности, соответствующий этой величине.

Бор [10] выступил с критикой этого утверждения. Он считал, что и после того как квантовые частицы $A$ и $B$ перестали взаимодействовать, они не могут рассматриваться как отдельные реальности. В этом смысле квантовая теория принципиально отличается от классической. В классической теории считается, что если объект пренебрежимо слабо взаимодействует с другими объектами, то он может изучаться как отдельная реальность. В квантовой теории Бора это не так.

Предложенный в настоящей статье подход к квантовой механике не противоречит требованиям, сформулированным в работе [2]. Фигурирующая в требовании 1 копия - 
это физическое состояние каждой из частиц. Реальность, которая фигурирует в требовании 2, будет существовать, если физическое состояние каждого квантового объекта однозначно определяется его материальной структурой. Такое предположение вполне укладывается в рамки предложенного подхода (см. [8]).

Таким образом, в предлагаемом подходе парадокс не возникает. При любом способе измерения спина у частицы $A$ физическое состояние частицы $B$ (объективная реальность) будет одним и тем же $\varphi \in\{\varphi\}_{-S_{z}(A)} \cap\{\varphi\}_{-} S_{x}(A)$. Различные квантовые состояния частицы $B$ связаны исключительно с субъективным выбором наблюдателем прибора для измерения наблюдаемой $\hat{A}$.

Из приведенного примера видно, что функционал $\varphi$ может рассматриваться в качестве специфического скрытого параметра. С одной стороны, конкретному событию отвечает определенный функционал $\varphi$. В этом смысле он является параметром. С другой стороны, с помощью эксперимента этот функционал однозначно зафиксировать нельзя, можно только установить его принадлежность к тому или иному ансамблю (квантовому состоянию). В этом смысле он является скрытым параметром.

После работ фон Неймана скрытые параметры приобрели “дурную репутацию” в квантовой механике. В своей знаменитой монографии [11] фон Нейман утверждает, что модели со скрытыми параметрами несовместимы с основными положениями квантовой механики. В качестве таких основных положений квантовой механики он принимает, в частности, аксиомы “а" и "б". Из этих аксиом он делает вывод, что любой квантово-механический ансамбль является дисперсионным, т.е. в этом ансамбле не все средние квадраты наблюдаемых равны квадратам средних соответствующих величин. В монографии [11] доказывается, что такой ансамбль нельзя разложить на бездисперсионные подансамбли, так как последние просто не сушествуют. Отсюда, в свою очередь, следует, что скрытые параметры ввести нельзя.

Однако в предлагаемом здесь подходе аксиомы “а" и “б” отсутствуют. Соответствующие утверждения справедливы не для наблюдаемых (элементов алгебры), а только для их представлений, которые порождаются специального вида функционалами (квантовыми средними). Для физических состояний рассуждения фон Неймана не справедливы. Физическое состояние - это бездисперсионный “подансамбль", состояший из одного элемента. Его существование не запрешено, поскольку "средние" значения наблюдаемых по такому ансамблю определяются нелинейным функционалом $\varphi$. В силу нелинейности функционала $\varphi$ физическое состояние не связано с каким-либо представлением алгебры наблюдаемых линейными операторами, и для них не могут быть сформулированы аксиомы "а", "б" и "в".

Фактически в монограффии [11] показано, что линейность состояния находится в конфликте с гипотезой о скрытых параметрах и причинностью. Отсюда фон Нейман сделал вывод, что на микроскопическом уровне причинности нет, а на макроскопическом уровне она появляется за счет усреднения по большому числу непричинных событий.

Сформулированный в данной работе подход позволяет разрешить этот конфликт прямо противоположным способом. Можно считать, что на уровне единичного микроскопического явления причинность есть, а линейность отсутствует. Линейность (кванто-

4 Теоретическая и математическая физика, т. 129, № 1, 2001 г. 
вого) состояния появляется за счет усреднения по квантовому ансамблю. Переход от единичного явления к квантовому ансамблю приводит к замене исходного детерминизма вероятностной интерпретацией.

Помимо рассуждений фон Неймана имеется еше один, не менее знаменитый аргумент против схем со скрытыми параметрами - это неравенство Белла [12]. Воспроизведем типичный вывод этого неравенства.

Пусть квантовый объект $Q$ (для простоты частица со спином 0) распадается на два объекта $A$ и $B$ (частицы со спинами $1 / 2$ ). Объекты $A$ и $B$ разлетаются на большое расстояние и попадают в детекторы $D(A)$ и $D(B)$, соответственно, измерения в которых независимы. Объект $A$ имеет набор наблюдаемых $\hat{A}_{a}$ (удвоенная проекция спина на направление $a$ ). Наблюдаемые, соответствующие разным значениям индекса $a$, не являются совместно измеримыми. Каждая из наблюдаемых может принимать два значения \pm 1 . В конкретном эксперименте прибор $D(A)$ измеряет наблюдаемую $\hat{A}_{a}$ с определенным индексом $a$. Для объекта $B$ все аналогично.

Предположим, что квантовый объект $Q$ имеет скрытый параметр $\lambda$. В каждом индивидуальном событии параметр $\lambda$ имеет определенное значение. Распределение событий по параметру $\lambda$ характеризуется вероятностной мерой $\mu(\lambda)$ с обычными свойствами

$$
\mu(\lambda) \geqslant 0, \quad \int d \mu(\lambda)=1
$$

Все величины, связанные с индивидуальным событием, зависят от параметра $\lambda$. В частности, значения наблюдаемых $\hat{A}_{a}$ и $\widehat{B}_{b}$, получаемые в конкретном эксперименте, являются функциями $A_{a}(\lambda), B_{b}(\lambda)$ параметра $\lambda$.

Для индивидуального события коррелящия наблюдаемых $\hat{A}_{a}$ и $\widehat{B}_{b}$ характеризуется величиной $A_{a}(\lambda) B_{b}(\lambda)$. Среднее значение этой величины определяется как корреляционная функция $E(a, b)$ :

$$
E(a, b)=\int d \mu(\lambda) A_{a}(\lambda) B_{b}(\lambda)
$$

Придавая индексам $a$ и $b$ различные значения и принимая во внимание, что

$$
A_{a}(\lambda)= \pm 1, \quad B_{b}(\lambda)= \pm 1
$$

получаем следующее неравенство:

$$
\begin{aligned}
\mid E(a, b) & -E\left(a, b^{\prime}\right)|+| E\left(a^{\prime}, b\right)+E\left(a^{\prime}, b^{\prime}\right) \mid \leqslant \\
& \leqslant \int d \mu(\lambda)\left[\left|A_{a}(\lambda)\right|\left|B_{b}(\lambda)-B_{b^{\prime}}(\lambda)\right|+\left|A_{a^{\prime}}(\lambda)\right|\left|B_{b}(\lambda)+B_{b^{\prime}}(\lambda)\right|\right]= \\
& =\int d \mu(\lambda)\left[\left|B_{b}(\lambda)-B_{b^{\prime}}(\lambda)\right|+\left|B_{b}(\lambda)+B_{b^{\prime}}(\lambda)\right|\right] .
\end{aligned}
$$

Согласно равенствам (6) для каждого значения $\lambda$ в правой части формулы (7) одно из выражений

$$
\left|B_{b}(\lambda)-B_{b^{\prime}}(\lambda)\right|, \quad\left|B_{b}(\lambda)+B_{b^{\prime}}(\lambda)\right|
$$


равно нулю, а другое - двум. Отсюда получаем неравенство Белла

$$
\left|E(a, b)-E\left(a, b^{\prime}\right)\right|+\left|E\left(a^{\prime}, b\right)+E\left(a^{\prime}, b^{\prime}\right)\right| \leqslant 2 .
$$

Корреляционная функция $E(a, b)$ легко вычисляется в рамках стандартной квантовой механики. В частности, если $A$ и $B$ - частицы со спином $1 / 2$, получим

$$
E(a, b)=-\cos \theta_{a b},
$$

где $\theta_{a b}$ - угол между направлениями $a$ и $b$. Легко подобрать такие направления $a, b, a^{\prime}, b^{\prime}$, для которых формулы (9) и (10) противоречат друг другу. Эксперимент свидетельствует в пользу формулы (10).

Казалось бы, этот вывод можно повторить в предлагаемом здесь подходе, сделав замены типа

$$
A(\lambda) \rightarrow \varphi(\hat{A}), \quad B(\lambda) \rightarrow \varphi(\widehat{B}), \quad \int d \mu(\lambda) \ldots \rightarrow \int_{\varphi \in\{\varphi\}_{Q}^{\hat{A} \widehat{B}}} d \mu(\varphi) \varphi(\ldots) .
$$

Однако это мнение ошибочно. Для вывода неравенства Белла существенно, что все слагаемые в левой части неравенства (7) можно представить в виде единого интеграла по параметру $\lambda$. Для заменяющего этот интеграл квантового среднего это несправедливо, так как в нем интегрирование нужно вести по актуальным состояниям. Фигурируюшие в разных корреляционных функциях элементы $\hat{A}_{a} \widehat{B}_{b}, \hat{A}_{a^{\prime}} \widehat{B}_{b}, \hat{A}_{a} \widehat{B}_{b^{\prime}}, \hat{A}_{a^{\prime}} \widehat{B}_{b^{\prime}}$ не коммутируют между собой. Поэтому равна нулю вероятность пересечения множеств актуальных состояний для этих элементов. При выводе неравенства (9) мы предполагали, что выражения (8) сушествуют для каждого $\lambda$. Однако вероятность сушествования физического состояния $\varphi$, которое было бы актуальным как для наблюдаемой $\widehat{B}_{b}$, так и для наблюдаемой $\widehat{B}_{b^{\prime}}$, равна нулю.

\section{5. ЗАКЛЮЧЕНИЕ}

Теперь обсудим, какое место предложенньй в данной статье подход к квантовой теории занимает среди других возможных подходов.

В любом современном подходе к квантовой теории основными структурными элементами являются наблюдаемые и состояния физической системы. В стандартной квантовой механике в качестве базового структурного элемента выступает гильбертово пространство. Наблюдаемые величины отождествляются с самосопряженными линейными операторами в этом пространстве, а состояния - либо с векторами (точнее, с лучами), либо со статистическими операторами (матрицами плотности).

В рамках стандартной квантовой механики удалось построить очень хорошо работающий математический аппарат. Поэтому возникает естественный вопрос: стоит ли пытаться строить квантово-теоретические модели, выходящие за рамки стандартной квантовой механики? 
Основания для таких попыток есть. Прежде всего, это проблема физической интерпретации. Гильбертово пространство - это весьма специфическое математическое понятие, которому непросто дать непосредственную физическую интерпретацию. Последовательное построение стандартной квантовой механики требует специальной “квантовой логики", которую также трудно физически интерпретировать. Все это приводит к тому, что до сих пор не утихают споры о последовательной интерпретации квантовой механики. Более того, в последние годы эти споры даже обострились.

Другим основанием для попыток выйти за рамки стандартной квантовой механики явились трудности, с которыми столкнулась квантовая теория при описании систем с бесконечным числом степеней свободы. Как показал фон Нейман, для систем с конечным числом степеней свободы все представления канонических перестановочных соотношений эквивалентны. В связи с этим стандартная квантовая механика фактически дает универсальное описание таких систем. Однако для систем с бесконечным числом степеней свободы такого утверждения сделать нельзя. Поэтому для их универсального описания требуется более обшая конструкция. Именно такая конструкция реализуется в алгебраическом подходе.

Существуют несколько вариантов алгебраического подхода. Однако во всех этих вариантах базовым понятием является алгебра наблюдаемых (глобальных или локальных). Несмотря на кажущуюся абстрактность этого понятия, алгебра наблюдаемых имеет достаточно простую физическую интерпретацию.

В качестве второго ингредиента алгебраического подхода принимается множество линейных положительных функционалов на алгебре наблюдаемых, которое отождествляется с множеством состояний физической системы. Эти функционалы имеют достаточно простую физическую интерпретацию. Постулируется, что значение этого функционала на элементе алгебры совпадает со средним значением наблюдаемой для физических систем, находящихся в соответствующем состоянии.

С одной стороны, рамки алгебраического подхода оказываются более широкими, чем рамки стандартной квантовой механики. Поэтому есть надежда, что в алгебраическом подходе хотя бы частично удастся обойти трудности, с которыми столкнулась стандартная квантовая механика.

С другой стороны, благодаря конструкции Гельфанда-Наймарка-Сигала в рамках алгебраического подхода можно получить основные ингредиенты стандартной квантовой механики - гильбертово пространство и линейные операторы. В рамках алгебраического подхода эти понятия оказываются не базовыми, а вторичными. При этом конструкция, которая используется в стандартной квантовой механике, оказывается одним из возможных неэквивалентных представлений алгебры наблюдаемых.

Таким образом, переход от стандартной квантовой механики к алгебраическому подходу - это переход к более глубокому уровню квантовой теории. В этом плане система аксиом, предлагаемая в настояшей статье, может рассматриваться как переход на еше более глубокий уровень.

Сформулированные в настояшей статье первые две аксиомы, касающиеся алгебры наблюдаемых, непосредственно позаимствованы из традиционного алгебраического под- 
хода. Однако далее наступает расхождение. В предлагаемом подходе выделенную роль играют действительные коммутативные подалгебры наблюдаемых. Именно с их помощью вводится (см. третий постулат) существенно новый элемент - нелинейный функционал $\varphi$, значения которого описывают возможный результат индивидуального измерения. Этому функционалу приписьвается смысл физического состояния. В отличие от него, состояние традиционного алгебраического подхода (линейный функционал) в настоящей статье называется квантовым состоянием.

Первичным элементом предлагаемого подхода является физическое состояние, а квантовое состояние оказывается вторичным элементом, классом эквивалентности физических состояний. В стандартной квантовой механике и традиционном алгебраическом подходе результат индивидуального измерения (физическая реальность) не имеет математической копии. В предлагаемом подходе такой копией является нелинейный функционал $\varphi$.

С одной стороны, функционал $\varphi$ имеет простую физическую интерпретацию. С другой стороны, этот функционал позволяет снабдить алгебру наблюдаемых структурой $C^{*}$-алгебры. Это дает возможность в дальнейшем воспользоваться конструкцией Гельфанда-Наймарка-Сигала и перейти к математической схеме стандартной квантовой механики. В традиционном алгебраическом подходе обычно постулируется, что алгебра наблюдаемых является $C^{*}$-алгеброй. С физической точки зрения это совсем не очевидно.

В стандартной квантовой механике и традиционном алгебраическом подходе постулируется, что функционал, ассоциированный с квантовым состоянием, описывает статистически среднее значение соответствуюшей наблюдаемой. В предлагаемом подходе этот функционал строится с помошью статистического усреднения функционалов $\varphi$. Поэтому он по построению описывает среднее значение наблюдаемой.

При аксиоматическом построении теории часто весьма непростым является вопрос о существовании нетривиальных моделей, удовлетворяюших сформулированным аксиомам. Известно, насколько болезненна эта проблема в аксиоматическом подходе к квантовой теории поля. Однако в рассматриваемом в настоящей статье случае эта проблема решается просто.

Стандартная квантовая механика представляет огромное количество таких моделей. Можно рассмотреть любую из них, например гармонический осциллятор. Для этой системы известны гильбертово пространство квантовых состояний и множество $\widetilde{\mathfrak{A}}_{+}$самосопряженных линейных операторов, которые соответствуют наблюдаемым. По линейности это множество расширяется до комплексной алгебры $\widetilde{\mathfrak{A}}$. Эта алгебра удовлетворяет всем постулатам, которым должна удовлетворять алгебра динамических величин $\mathfrak{A}$.

Структура множеств $\widetilde{\mathfrak{A}}$ и $\widetilde{\mathfrak{A}}_{+}$полностью известна. Поэтому в принципе мы можем найти все максимальные действительные коммутативные подалгебры. В этих подалгебрах выберем системы образующих с помощью процедуры построения координат в фазовом пространстве. Далее найдем спектры всех образующих. По точкам этих спектров, используя свойство (2д), построим функционалы $\tilde{\varphi}$, сужение которых на каждую 
максимальную подалгебру реализует действительный гомоморфизм. Эти функционалы будут обладать свойствами физических состояний $\varphi$. Таким образом, мы можем построить множество всех физических состояний для данной физической системы.

Построить вероятностную меру на этом пространстве, точнее на его подпространствах, соответствующих каждому квантовому состоянию $\Psi_{Q}$, можно следующим образом. Вероятность обнаружить значение $A$ наблюдаемой $\hat{A}$ в состоянии $\Psi_{Q}$ вычислим по формулам стандартной квантовой механики, используя соответствующие векторы гильбертова пространства. Конструкция Гельфанда-Наймарка-Сигала гарантирует допустимость этой операции. После этого вычислим вероятность обнаружить все значения наблюдаемой $\hat{A}$, которые удовлетворяют условию $A \leqslant \tilde{A}$. Эта вероятность будет вероятностной мерой $\mu_{Q}(\varphi)\left(\varphi(\hat{A}) \leqslant \tilde{A}, \varphi \in\{\varphi\}_{Q}^{\hat{A}}\right)$.

В настоящей статье рассмотрены только кинематические и статистические проблемы квантовой механики. Это позволило обойтись без предположений о виде взаимодействия.

В предлагаемую здесь схему квантовой механики может быть встроена динамика, которая описывает не только унитарную временну́ю эволюцию квантовых ансамблей (взаимодействие второго рода по терминологии фон Неймана [11]), но и взаимодействие с измерительным прибором (взаимодействие первого рода). В рамках этой динамики могут быть объяснены корпускулярно-волновой дуализм и коллапс квантового состояния.

Вариант такой динамики описан в статье [8]. Однако для ее построения требуется делать более детальные предположения о строении квантового объекта. Эти предположения носят значительно менее общий характер, чем те, которые сделаны в данной статье. Они могут показаться гораздо более спорными.

\section{Список литературы}

[1] A. Einstien. J. Franklin Institute. 1936. V. 221. P. 349.

[2] A. Einstein, B. Podolsky, N. Rosen. Phys. Rev. 1935. V. 47. P. 777.

[3] Ж. Эмх. Алгебраический подход в статистической механике и квантовой теории поля. М.: Мир, 1976.

[4] C. С. Хоружий. Введение в алгебраическую квантовую теорию поля. М.: Наука, 1986.

[5] Н. Н. Боголюбов, А. А. Логунов, А.И. Оксак, И. Т. Тодоров. Общие принципы квантовой теории поля. М.: Наука, 1987.

[6] У. Рудин. Функциональный анализ. М.: Мир, 1975.

[7] Г. А. Корн, Т. Корн. Справочник по математике для научных работников и инженеров. М.: Наука, 1984.

[8] D. A. Slavnov. Causality and probabilistic interpretation of quantum mechanics. quant-ph/ 0001061.

[9] Д. Бом. Квантовая теория. М.: Наука, 1965.

[10] Н. Бор. УФН. 1936. Т. 16. С. 446.

[11] И. фон Нейман. Математические основы квантовой механики. М.: Наука, 1964.

[12] J. S. Bell. Physics (New York). 1965. V. 1. P. 195. 